\title{
Parallel FoxP1 and FoxP2 Expression in Songbird and Human Brain Predicts Functional Interaction
}

\author{
Ikuko Teramitsu, ${ }^{1}$ Lili C. Kudo, ${ }^{2}$ Sarah E. London, ${ }^{2}$ Daniel H. Geschwind, ${ }^{2,4}$ and Stephanie A. White ${ }^{1,2,3}$ \\ Interdepartmental Programs in ${ }^{1}$ Molecular, Cellular, and Integrative Physiology, and ${ }^{2}$ Neuroscience, ${ }^{3}$ Department of Physiological Science and the Brain \\ Research Institute, and ${ }^{4}$ Department of Neurology and Neurogenetics Program in the David Geffen School of Medicine, University of California, Los \\ Angeles, California 90095
}

\begin{abstract}
Humans and songbirds are two of the rare animal groups that modify their innate vocalizations. The identification of FOXP2 as the monogenetic locus of a human speech disorder exhibited by members of the family referred to as KE enables the first examination of whether molecular mechanisms for vocal learning are shared between humans and songbirds. Here, in situ hybridization analyses for FoxP1 and FoxP2 in a songbird reveal a corticostriatal expression pattern congruent with the abnormalities in brain structures of affected KE family members. The overlap in FoxP1 and FoxP2 expression observed in the songbird suggests that combinatorial regulation by these molecules during neural development and within vocal control structures may occur. In support of this idea, we find that FOXP1 and FOXP2 expression patterns in human fetal brain are strikingly similar to those in the songbird, including localization to subcortical structures that function in sensorimotor integration and the control of skilled, coordinated movement. The specific colocalization of FoxP1 and FoxP2 found in several structures in the bird and human brain predicts that mutations in FOXP1 could also be related to speech disorders.
\end{abstract}

Key words: forkhead; language; song; speech; vocal learning; zebra finch

\section{Introduction}

The neural mechanisms for auditory-guided vocal learning are incompletely understood. Surprisingly, vocal learning (i.e., the ability to substantially modify innate vocalizations to mimic a vocal model) is evident in only a few animal groups, including songbirds and humans but not other primates or rodents (Snowdon and Hausberger, 1997; Doupe and Kuhl, 1999). In songbirds, the development and production of learned song is subserved by interconnected regions of the pallium (also called cortical mantle), striatum, and thalamus, collectively known as the song circuit (Fig. 1) (Bottjer and Johnson, 1997; Farries, 2001). Identification of the molecules that define and operate within this circuit would provide insight into the neural mechanisms for song learning, enable comparison to humans, and possibly reveal shared mechanisms for vocal learning. For example, a molecule known as synelfin in songbirds and $\alpha$-synuclein in mammals is regulated in song circuitry during song learning and has been linked to

\footnotetext{
Received Sept. 22, 2003; revised Jan. 23, 2004; accepted Jan. 26, 2004.

This work was supported by a Medical Investigation of Neurodevelopmental Disorders (University of California, Davis) scholarship (I.T.), by Grant MH-60233 (D.H.G. and L.C.K.), and by the National Alliance for Autism Research, the Alfred P. Sloan Foundation, and the Mental Retardation Research Center at the University of California, Los Angeles (S.A.W.). Human fetal tissue was obtained from the University of Maryland Brain and Tissue bank, which is funded by a grant from the National Institutes of Health. We thank Paige C. Nilson and Amy Poopatanapong for technical assistance, other members of the White laboratory for helpful discussion, and the Arnold and Schlinger laboratories for sharing resources. Drs. A. Arnold, F. Schweizer, and A. Silva, and two anonymous reviewers provided helpful comments on the present or previous versions of this manuscript. Drs. W. Grisham, T. Preuss, and J. M. Wild lent insight to anatomical findings.

Correspondence should be addressed to Dr. Stephanie A. White, 621 Charles E. Young Drive South, Los Angeles, CA 90095-1606. E-mail: swhite@physci.ucla.edu.

DOI:10.1523/JNEUROSCI.5589-03.2004

Copyright $\odot 2004$ Society for Neuroscience $\quad$ 0270-6474/04/243152-12\$15.00/0
}

Parkinson's and Alzheimer's diseases in humans (Clayton and George, 1999). Such findings indicate that common mechanisms may underlie specific motor and memory processes in birds and humans.

Recently, FOXP2, which encodes a member of the Forkhead box (Fox) family of proteins, has been identified as the gene underlying a human developmental language abnormality (Lai et al., 2001). FOX proteins are transcriptional regulators characterized structurally by a DNA-binding domain that forms a winged helix and functionally as embryonic morphogenerators (Carlsson and Mahlapuu, 2002) [See Kaestner et al. (2000) and http:// www.biology.pomona.edu/fox.html for nomenclature. Briefly, nucleotide sequences are italicized whereas proteins are not. Human forms are capitalized (e.g. FOXP2 protein), murine forms are in lowercase (e.g. Foxp2), and those of other species, such as the zebra finch, are in uppercase and lowercase (e.g. FoxP2).] The FOXP subfamily has four members and is distinguished by a divergent winged-helix domain and a novel zinc finger motif (Shu et al., 2001; Lu et al., 2002). Whereas Foxp3 is expressed in T-cells (O'Garra and Vieira, 2003), Foxp1, Foxp2, and Foxp4 are implicated in lung development. They are also expressed in brain (Shu et al., 2001; Lu et al., 2002; Ferland et al., 2003; Takahashi et al., 2003). However, no neural role had been hypothesized for these molecules before discovery of the human mutation in FOXP2.

Individuals with a FOXP2 mutation exhibit prominent deficits in orofacial movements, called buccal-oral apraxia, but perform normally for simple oral and limb movements (VarghaKhadem et al., 1998; Alcock et al., 2000a; Watkins et al., 2002a). They are impaired on tests of verbal fluency and language com- 
A
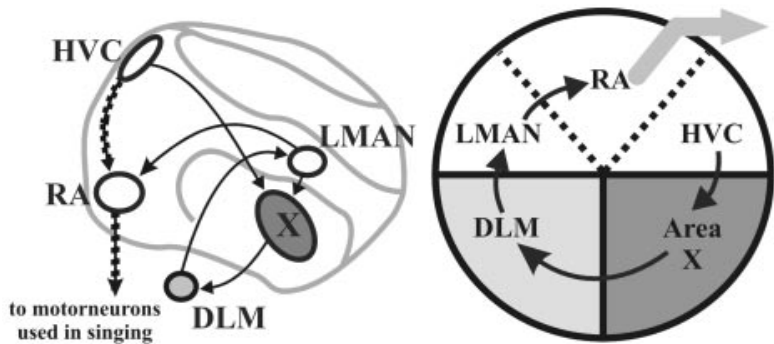

B Human circuits

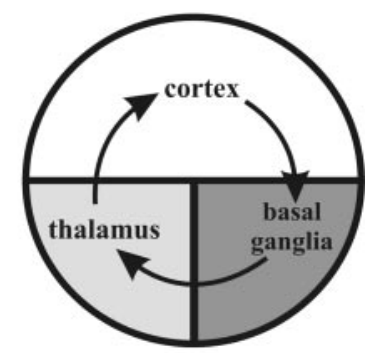

Figure 1. Schematic views of the avian song circuit and human cortico-basal ganglia-thalamo-cortical circuitry. The cortex is white, basal ganglia dark gray, and thalamus is light gray. A, Left, Composite sagittal view of songbird telencephalon. Auditory input (not shown) enters the song circuit at the HVC, the neurons of which contribute to two pathways. The vocal motor pathway (stippled arrows) controls song production and is composed, sequentially, of the hyperpallial nucleus HVC, the arcopallial nucleus RA, and brainstem motor neurons that innervate the song organ and respiratory muscles (data not shown) (Nottebohm et al., 1976; Wild, 1993). The anterior forebrain pathway APF; (plain arrows), which allows song modification (Bottjer et al., 1984; Scharff and Nottebohm, 1991; Williams and Mehta, 1999; Brainard and Doupe, 2000), begins with a subset of HV( neurons that project to area $X$ in the striatum (Mooney, 2000). The pathway proceeds through the DLM in the thalamus, back to the pallial nucleus LMAN. Projections of LMAN neurons join the two pathways at RA (Nottebohm et al., 1982; Okuhata and Saito, 1987; Bottjer et al., 1989; Mooney and Konishi, 1991), and these same LMAN neurons send axon collaterals back to area X (Vates and Nottebohm, 1995). Middle, Schematic focuses on the AFP, a cortico-striato-thalamo-cortical circuit. In this simplified scheme, LMAN to area $X$ connections, among others, are not shown. The gray arrow indicates telencephalic output onto motor neurons. $B$, Schematic of human cortico-basal ganglia-thalamo-cortical circuitry for comparison.

prehension, in addition to language production (Marcus and Fisher, 2003). These behavioral features are accompanied by structural abnormalities in the cortex and striatum among other brain regions, and atypical activity of a corticostriatal network that participates in both covert and overt speech (Lai et al., 2001; Belton et al., 2003; Liegeois et al., 2003). Although the affected phenotype is not limited to language, linguistic difficulties are prominent (Vargha-Khadem et al., 1995), indicating that FOXP2 lies along one neural pathway linked to language. A conservative interpretation of the behavioral and neuroanatomical profiles of affected individuals suggests a core deficit in complex coordinated orofacial movements, including speech, that require procedural learning (Packard and Knowlton, 2002; Watkins et al., 2002a; Marcus and Fisher, 2003). This makes evolutionary study of FOXP2 of great interest, particularly with reference to the capacity for vocal learning (Doupe and Kuhl, 1999).

We, therefore, identified the complete cDNA sequence for the songbird FoxP2 homolog using the zebra finch, Taeniopygia guttata. In this species, only males learn to sing a courtship song. The brain regions that comprise the song circuit are much smaller or lacking in females (Nottebohm and Arnold, 1976). We also investigated FoxP1 because it is the closest forkhead family member to FoxP2, shares similar domains whereby it represses transcription of genes that are also affected by FoxP2, and can dimerize with FoxP2 (Shu et al., 2001; Wang et al., 2003; Li et al., 2004).

We found cortical, striatal, and thalamic expression of both genes, including overlapping expression in some song nuclei. FoxP1 exhibited a striking sexual dimorphism, nearly concordant with the sexual dimorphism of the song circuit. These discoveries in zebra finch brain motivated us to examine FOXP1 and FOXP2 in human brain, in which expression was again found to partially overlap in striatal and thalamic structures. This unique comparative approach between songbird and human implicates both genes in the formation and function of circuitry that uses sensory feedback to learn voluntary, sequential, orofacial gestures.

\section{Materials and Methods}

Animals and tissues. All animal use was approved by the University of California, Los Angeles Institutional Animal Care and Use Committee.

Total and poly $\left(\mathrm{A}^{+}\right)$RNA isolation and in situ hybridization analyses were performed on tissues from $>150 \mathrm{~d}$ after hatching $(>\mathrm{d} 150), \mathrm{d} 40$, $\mathrm{d} 35$, and d1 zebra finches. After decapitation, tissues were dissected rapidly, frozen on aluminum floats on liquid nitrogen, and stored at $-80^{\circ} \mathrm{C}$, except for in situ analysis of $\mathrm{d} 1$ brains in which the whole head was immersed in cold embedding medium, optimal cutting temperature (Sakura Finetek, Torrance, CA), and frozen in methanol with dry ice (Perlman et al., 2003). Total RNA was isolated from $d 40$ brain and heart using the RNeasy Mini kit (Qiagen, Valencia, CA). Poly $\left(\mathrm{A}^{+}\right)$RNA was isolated from $\mathrm{d} 40$ brain using the Oligotex mRNA Maxi kit (Qiagen).

Human tissues. Human tissue was obtained from the Brain and Tissue Bank for Developmental Disorders at the University of Maryland (Baltimore, MD). Gestational age was estimated based on the mother's last menstrual period. After fetal extraction, the tissue was frozen rapidly on dry ice and stored at $-80^{\circ} \mathrm{C}$ before sectioning. Postmortem intervals ranged between 0.1 and $3 \mathrm{hr}$. Tissue from four fetal brains was used. Human fetal stages were chosen to correspond with the time when neurogenesis for subcortical structures, such as the basal ganglia and thalamus, is mostly complete, when the tissue quality for RNA studies is high enough to be comparable to experimental animals (short postmortem interval), and when the brain is small enough to be assessed in a single large slide.

Isolation of zebra finch FoxP1 and FoxP2 cDNAs. Oligo(dT)-primed total RNA was reverse transcribed to cDNA using Superscript II reverse transcriptase (Invitrogen, Carlsbad, CA). Partial cDNAs were first amplified by PCR using degenerate primers designed to hybridize with the zinc finger and Fox domains in mouse and human FOXP1 and FOXP2 (sense 5'MGRGTDCAAATGCARGTKGT- $3^{\prime}$; antisense 5'-TGMCGBACTG CRTTCTTCCA-3'). A FoxP1 cDNA fragment $3^{\prime}$ to the obtained segment was then isolated from the heart and brain using a sense primer specific to zebra finch FoxP1 (5'-CTGGTTCACACGAATGTTCGC-3') and a degenerate antisense primer ( $5^{\prime}$-CACTCCATGTCCTCRTTTACTG-3'). This $3^{\prime}$ FoxP1 coding fragment revealed the zebra finch-specific sequence that was then used to obtain the first probe for in situ hybridization (see below). The entire ORF of FoxP2 was subsequently obtained from brain cDNA reverse transcribed from poly $\left(\mathrm{A}^{+}\right)$RNA using the Marathon cDNA amplification kit (Becton Dickinson Biosciences, San Jose, CA) with primers based on consensus sequences within mouse (accession number AY079003) and human (accession number AF337817) Foxp2 5' and 3' untranslated regions (UTRs) (sense $5^{\prime}$-AGAGAAAGGTATTAAGTC-3'; antisense 5'-GCTTAGTAAGTTCCTTTAGGG-3'). PCR cycling conditions using the Advantage cDNA PCR kit (Becton Dickinson Biosciences) were: (1) FoxP1/Foxp2 fragments with consensus degenerate primers $\left(2 \mathrm{~min}\right.$ at $94^{\circ} \mathrm{C}$ for $1 \mathrm{cycle} ; 15 \mathrm{sec}$ at $94^{\circ} \mathrm{C}, 30 \mathrm{sec}$ at $58^{\circ} \mathrm{C}$, and 1 min at $72^{\circ} \mathrm{C}$ for 35 cycles; and $30 \mathrm{sec}$ at $72^{\circ} \mathrm{C}$ for 1 cycle); (2) FoxP1 $3^{\prime}$ fragment [as described under (1), except for the annealing temperature $57^{\circ} \mathrm{C}$ ); and (3) full-length FoxP2 $\left(2 \mathrm{~min}\right.$ at $94^{\circ} \mathrm{C}$ for 1 cycle; $15 \mathrm{sec}$ at $94^{\circ} \mathrm{C}$, $30 \mathrm{sec}$ at $46^{\circ} \mathrm{C}, 4 \mathrm{~min}$ at $72^{\circ} \mathrm{C}$ for 35 cycles; and $3 \mathrm{~min}$ at $72^{\circ} \mathrm{C}$ for 1 cycle).

Amplified cDNAs were subcloned into pCR 4-TOPO vector (Invitrogen) and sequenced in sense and antisense directions. For the full-length FoxP2 cDNA, a total of 15 independent subclones were sequenced. For the two FoxP1 fragments, two subclones were sequenced for the first fragment and 10 subclones for the second, more 3', fragment.

Zebra finch probe synthesis. Two distinct regions each from FoxP1 and FoxP2 were chosen for generating probes for in situ hybridization analyses to ensure the specificity of the expression patterns observed for each gene. The first probes were designed to hybridize to the $3^{\prime}$ portion of FoxP1 and FoxP2, respectively. For FoxP1, this was the region corresponding to 1708-2011 bp of human FOXP1 (accession number NM_ 032682 ) relative to the start codon. For FoxP2, this was $1870-2127$ bp of 
the newly cloned zebra finch FoxP2 relative to the start codon. cDNA fragments coding these regions were amplified by PCR with primers designed from zebra finch FoxP1 and FoxP2 sequences, respectively (FoxP1: sense 5'-AATGCTGCTTTACAGGCT-3', antisense 5'-GTTCATCTTCATAATCTCTG-3'; FoxP2: sense 5' -ATAAATAACGCATCCAGTGGC3', antisense 5'-TTCCAGATCTTCAGATAAAGGC-3'). Cycling conditions were as mentioned above.

The second probes were designed to hybridize to the coding region upstream of the zinc finger domain of each of the genes (FoxP1: region corresponding to 661-998 bp of human FOXP1, accession number NM 032682; FoxP2: 676-1005 bp of zebra finch FoxP2, accession number AY395709). Of note, if the zebra finch possesses variant FoxP transcripts, as observed in the mouse (Shu et al., 2001), this second probe should recognize multiple forms. FoxP1 and FoxP2 cDNA fragments coding these second probe regions were amplified by PCR using the Advantage 2 PCR kit (Becton Dickinson Biosciences) with primers designed from chicken FoxP1 (accession number BQ 038849) and zebra finch FoxP2 sequences, respectively (FoxP1: sense 5' -CAAGGCATGATTCCAACAGAACTGC-3', antisense 5' AGCGCATGCTCACTGTTGAGATG-3'; FoxP2: sense 5' -CATCTGCTGAACCTTCAGCG-3', antisense 5'-AGTATGGGAGGCCCCAGTCT-3'). Cycling conditions for both primer sets were: (1) $1 \mathrm{~min}$ at $95^{\circ} \mathrm{C}$ for $1 \mathrm{cycle}$; (2) $20 \mathrm{sec}$ at $95^{\circ} \mathrm{C}, 1 \mathrm{~min}$ at $68^{\circ} \mathrm{C}$ for 35 cycles; (3) $1 \mathrm{~min}$ at $68^{\circ} \mathrm{C}$ for 1 cycle; and (4) $10 \mathrm{~min}$ at $70^{\circ} \mathrm{C}$ for 1 cycle.

Amplified fragments were subcloned into pCR4-TOPO vector (Invitrogen), sequenced to reconfirm their identity, and then used for in vitro transcription to generate sense and antisense RNA probes labeled with $\left[{ }^{33} \mathrm{P}\right]$ UTP (Amersham Biosciences, Piscataway, NJ) using Riboprobe Combination System-T3/T7 (Promega, Madison, WI).

Human probe synthesis. cDNA from the left temporal cortex of a 19 week human fetal brain was obtained as follows: total RNA was extracted using TriZol (Invitrogen) according to the manufacturer's recommendations, followed by first-strand cDNA synthesis with SuperScript II FirstStrand Synthesis System for RT-PCR (Invitrogen). RT-PCR was performed with primers designed from FOXP1 and FOXP2 sequences, respectively (FOXP1: sense 5'-GCCGATTCATTCCACGCAGCAGT A-3', antisense 5' CCACACCC GTTATCGCAGAGCAC-3'; FOXP2: sense 5'- CCACGA AGACCTCAATGGTT-3', antisense 5' -TCACGCTGAGGTTTCACAAG$\left.3^{\prime}\right)$. Cycling conditions for both primer sets were: (1) $1 \mathrm{~min}$ at $94^{\circ} \mathrm{C}$ for 1 cycle; (2) 2 min at $94^{\circ} \mathrm{C}, 45 \mathrm{sec}$ at $55.5^{\circ} \mathrm{C}$, and $1 \mathrm{~min}$ at $72^{\circ} \mathrm{C}$ for 33 cycles; and (3) $10 \mathrm{~min}$ at $72^{\circ} \mathrm{C}$ for $1 \mathrm{cycle}$. The FOXP1 probe sequence corresponds to 3413-3676 bp of NM_032682.3. The FOXP2 probe sequence corresponds to 2210-2462 bp of NM_148900.1. These probe sequences were chosen such that they did not find FOX genes other than the intended target when searched using the Basic Local Alignment Search Tool. PCR products were purified using QIAquick PCR purification kit (Qiagen) and cloned into pCRII-TOPO vector using the TOPO TA Cloning kit (Invitrogen).

In situ hybridization analyses of zebra finch. Analysis of FoxP gene expression was performed essentially as described by Jacobs et al. (1999), except that frozen sections were thaw-mounted on Superfrost Plus microscope slides (Fisher Scientific, Pittsburgh, PA) and postfixed with $4 \%$ paraformaldehyde, $\mathrm{pH}$ 7.4. Briefly, a series of $20 \mu \mathrm{m}$ thick coronal or sagittal sections were hybridized with $\left[{ }^{33} \mathrm{P}\right]$ UTP-labeled RNA probes. Five sets of slides containing adjacent sections were used, a set each for FoxP1 sense, antisense and FoxP2 sense, antisense probes. Equivalent counts per minute of sense and antisense probes for both FoxP1 and FoxP2 were loaded per slide. The fifth set was stained with thionin (Tolivia and Tolivia, 1985) to enable identification of neuroanatomical structures and to guide localization of the expression patterns for each gene with reference to a songbird brain atlas (Stokes et al., 1974) (Table 1 ). Wherever possible, specific structures were named, but when the anatomy was less clear, as in d1 bird brains, more general descriptions were used. After hybridization, slides were apposed to autoradiographic film (BioMax MR film; Eastman Kodak, Rochester, NY) for 24-48 hr or $48-72 \mathrm{hr}$ for FoxP1 or FoxP2, respectively. Slides were then dipped in liquid emulsion (NTB-2; Eastman Kodak) and exposed at $4^{\circ} \mathrm{C}$ for 4 or 5 weeks, for FoxP1 or FoxP2, respectively. Emulsion-coated slides were developed, dehydrated, and coverslipped for determination of expression patterns.

Several criteria were applied to assign the observed radioactive signals

\section{Table 1. Neuroanatomical abbreviations}

\begin{tabular}{|c|c|}
\hline Abbreviation & Region \\
\hline \multicolumn{2}{|c|}{ Songbird terminology } \\
\hline Area $X$ & Song nucleus within the striatum mediale \\
\hline DLM & Nucleus dorsolateralis anterior thalami, pars medialis \\
\hline DTZ & Dorsal thalamic zone \\
\hline GP & Globus pallidus \\
\hline HA & Hyperpallium apicale \\
\hline HD & Hyperpallium densocellulare \\
\hline LMAN & Lateral magnocellular nucleus of the anterior nidopallium \\
\hline M & Mesopallium \\
\hline MLd & Nucleus mesencephalicus lateralis, pars dorsalis \\
\hline $\mathrm{N}$ & Nidopallium \\
\hline Ov & Nucleus ovoidalis \\
\hline RA & Nucleus robustus arcopallialis \\
\hline RPgc & Nucleus reticularis pontis caudalis, pars gigantocellularis \\
\hline Rt & Nucleus rotundus \\
\hline SN & Substantia nigra \\
\hline StL & Striatum laterale \\
\hline StM & Striatum mediale \\
\hline SPC & Nucleus superficialis parvocellularis \\
\hline SpL & Nucleus spiriformis lateralis \\
\hline $\mathrm{Te} 0$ & Tectum opticum \\
\hline VTA & Ventral tegmental area \\
\hline \multicolumn{2}{|c|}{ Human terminology } \\
\hline Adl & Nucleus anterior thalami, dorsal \\
\hline Avl & Nucleus anterior thalami, ventral \\
\hline Caud & Nucleus caudatus \\
\hline CM & Nucleus centrum medianum thalami \\
\hline$C P$ & Cortical plate \\
\hline GPi & Globus pallidus, pars interna \\
\hline IZ & Intermediate zone \\
\hline MD & Nucleus medialis dorsalis thalami \\
\hline MZ & Marginal zone \\
\hline$P$ & Putamen \\
\hline Pcn & Nucleus paracentralis thalami \\
\hline Pf & Nucleus parafascicularis thalami \\
\hline $\mathrm{Rn}$ & Nucleus ruber \\
\hline SP & Subplate \\
\hline Stn & Nucleus subthalamicus \\
\hline VA & Nucleus ventralis anterior thalami \\
\hline VB(VPL/VPM) & Nucleus ventralis posterior lateralis/medialis thalami \\
\hline VL & Nucleus ventralis lateralis thalami \\
\hline VLc & Nucleus ventralis lateralis caudalis \\
\hline VM & Nucleus ventralis medialis thalami \\
\hline
\end{tabular}

to specific neuroanatomical regions (the latter were identified by Nissl stains and by reference to an atlas, as mentioned above). For each anatomical designation: (1) signals were detected by each of two nonoverlapping probes for a given gene; (2) signals were observed in consecutive sections; (3) similar expression patterns occurred across multiple birds ( $n \geq 3$ per age); (4) signals from film and emulsion-dipped sections corresponded; and (5) signals were detected with the antisense, but not with the sense, probes.

In situ hybridization analyses of human brains. In situ hybridization analyses of FOXP genes in human brain tissue were performed essentially as described by Geschwind et al. (2001) for human tissues, except that 20 $\mu \mathrm{m}$ thick coronal and sagittal sections were thaw-mounted onto $50 \times 70$ $\mathrm{mm}$ slides (Brain Research Laboratories, Newton, MA). These were air dried and postfixed in $4 \%$ buffered paraformaldehyde, $\mathrm{pH} 7.4$, for $20 \mathrm{~min}$ at room temperature, rinsed in $0.1 \mathrm{M}$ phosphate buffer and water, and air dried for $30 \mathrm{~min}$. Sections were stored dessicated at $-80^{\circ} \mathrm{C}$ before use. Briefly, before hybridization, slides were treated with glycine and acetic anhydride and TEA, followed by two $2 \times$ SSC washes and a series of ethanol washes. Hybridized slides were incubated overnight at $60^{\circ} \mathrm{C}$. The slides were then washed twice in $4 \times$ SSC at $60^{\circ} \mathrm{C}$, treated with RNase $\mathrm{A}$ in $45^{\circ} \mathrm{C}$, washed four times in $2 \times \mathrm{SSC}$ at room temperature, twice in $0.5 \times$ 


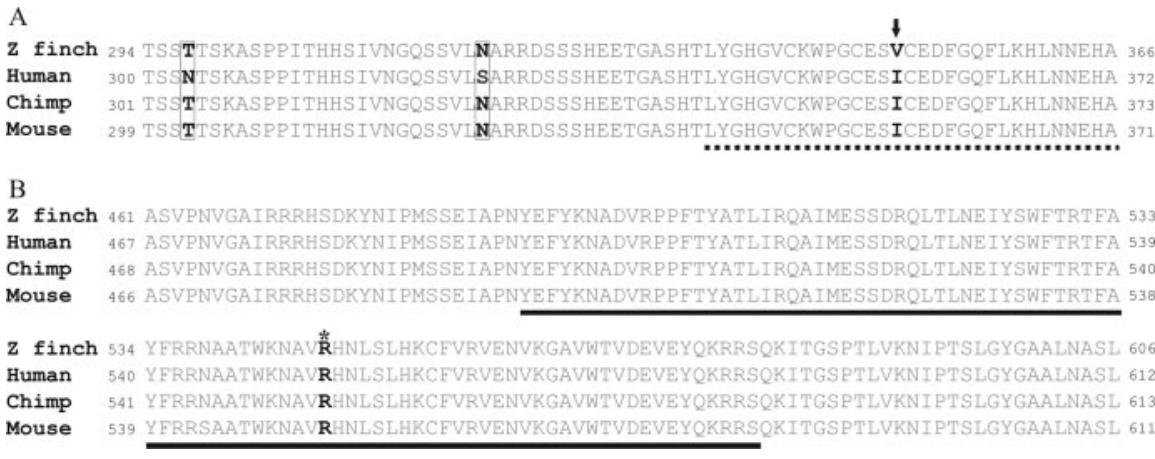

Figure 2. Alignment of deduced amino acid sequences from the zebra finch FoxP2 CDNA (GenBank accession number AY395709) with three mammalian sequences (accession numbers: AF337817, human; AF512947, chimpanzee; AF339106, mouse). $A$, The selected region includes the positions at which two residues in the human sequence (boxes; N303 and S325) differ from other primates (Enard et al., 2002). The putative zinc finger domain (dotted underscore) is also shown. In the zebra finch, a conservative substitution of valine for isoleucine at position 350 (arrow; I350V) occurs within this domain. Four additional zebra finch substitutions (S42T, S78G, S229N, and A243S; data not shown) occur at positions outside of the currently identified protein domains. B, Selected region spans the Fox domain (solid underscore) that shows 100\% identity between finch, human, and chimp. The asterisk indicates an invariant arginine at position 553 in humans that is mutated to histidine in a rare speech and language disorder (Lai et al., 2001).

SSC at $60^{\circ} \mathrm{C}$, once in $0.1 \times \mathrm{SSC}$ at $60^{\circ} \mathrm{C}$, once in $0.1 \times \mathrm{SSC}$ at room temperature, then rinsed in water. After hybridization, slides were apposed to autoradiographic film (BioMax MR film; Eastman Kodak) for a period of $5 \mathrm{~d}$, after which the film was developed under standard conditions. Control sections incubated with sense RNA showed no specific hybridization. Slides were then defatted in a series of ethanol and chloroform, dipped in NTB-2 emulsion (Eastman Kodak), and stored at $4^{\circ} \mathrm{C}$ for 4-5 weeks. Slides were developed in D-19 developer (Eastman Kodak) and fixed in Kodak Fixer both at $15^{\circ} \mathrm{C}$. After development, slides were stained with cresyl violet, followed by a series of ethanols, left in Citrisolv (Fisher Scientific) overnight, then coverslipped with Permount (Fisher Scientific).

Analysis of human brain structures labeled with cRNA probes was performed on both autoradiograms and emulsion-dipped slides stained with cresyl violet for optimal anatomical resolution. The use of a combination filtered bright field with dark-field epi-illumination (Darklite; Nikon, Melville, NY) allowed for simultaneous visualization of silver grains and cresyl violet-stained cells, facilitating structural analyses. Brain structures, including specific thalamic nuclei, were identified and labeled according to two reference atlases for primate embryonic brain and thalamus (Olszewski, 1952; Jones, 1985; Feess-Higgins and Larroche, 1987), with additional discussion with Dr. Todd Preuss (Emory University, Atlanta, GA), an expert primate comparative anatomist.

\section{Results}

\section{FoxP2 sequence in an avian vocal learner}

Among the identified primate FoxP2 sequences, human FOXP2 bears unique residues at positions 303 and 325 (Fig. 2). During evolution, these substitutions are posited to be key molecular events that gave rise to language, or minimally to the capacity for selection and sequencing of orofacial movements required for speech (Enard et al., 2002; Zhang et al., 2002; Clark et al., 2003). To discover whether songbirds, being vocal learners, possess the human substitutions, we cloned the full-length FoxP2 sequence from the zebra finch using primers designed based on consensus sequences of the mouse and human $5^{\prime}$ and $3^{\prime}$ UTRs. The complete cDNA encodes a predicted protein of 709 amino acids that contains a poly-glutamine tract (aa 152-225; data not shown), as well as the putative zinc finger domain (aa 336-366) and the Fox domain (aa 489-579), which are characteristic features of the FoxP subfamily (Shu et al., 2001). Regions of the zebra finchdeduced amino acid sequence are aligned with those from human, chimp, and mouse in Figure 2. The zebra finch sequence demonstrates $97 \%$ and $100 \%$ identities with the human homolog in the zinc finger and Fox domains, respectively. Of note, all amino acids shared between the two vocal learners, zebra finch and human, are shared with nonhuman primates as well. Because the zebra finch does not possess the humanspecific residues, yet can modify its vocalizations, it appears that no single FoxP2 sequence accounts for all instances of this behavioral trait. Intriguingly, zebra finch FoxP2 possesses five residues that are distinct from residues at the corresponding position in all mammalian homologs identified thus far (Fig. 2).

\section{FoxP2 in both sensory and motor structures of adult male zebra finch brain}

The structural and functional deficits observed in the cortex and striatum of humans bearing a FOXP2 mutation suggested that FOXP2 expression would be localized to these regions (Vargha-Khadem et al., 1998; Liegeois et al., 2003; Watkins et al., 2002b; Fisher et al., 2003). In songbirds, a cortico-striato-thalamo-cortical loop underlies the development and production of learned song (Fig. 1A) (for review, see Bottjer and Johnson, 1997; Farries, 2001). To determine whether the song circuit expresses FoxP2, we performed in situ hybridization on brain sections from adult male zebra finches $(>\mathrm{d} 150)$ whose neural structures are fully developed. In the telencephalon, FoxP2 is expressed at low levels in pallial (cortical) regions and high levels in the striatum, as hypothesized based on the structural and functional abnormalities of afflicted humans. There is substantial diencephalic expression and specific expression in some mesencephalic structures (Fig. 3-5). Interestingly, in addition to motor structures, FoxP2 is expressed in visual and auditory processing regions consistent with a potential role in sensory feedback.

The recent renaming of the avian brain facilitates meaningful comparisons with other vertebrates and indicates that pallial regions of the avian telencephalon are broadly homologous to the mammalian neocortex, claustrum, and pallial amygdale (Reiner et al., 2004). Within adult zebra finch telencephalon, FoxP2 is broadly expressed at low levels in the hyperpallium densocellulare (HD) and mesopallium and at even lower levels in the nidopallium (Fig. 3A). The specificity of this label is indicated by the comparative lack of signal in the arcopallium (Fig. 4A), in field L of the nidopallium (Fig. 5D), and, by comparison, with a sense control from an adjacent section (Fig. $3 A$ ). The song nucleus HVC (used as a proper name) is labeled, but not above the level of the surrounding nidopallium (Fig. 5A), as confirmed by visual inspection of emulsion-dipped slides (data not shown). The song nucleus lateral magnocellular nucleus of the anterior nidopallium (LMAN) shows signals at or below the level of the surrounding nidopallium (Figs. $3 A, 5 D$ ). As mentioned above, the arcopallium, including the premotor song nucleus, robustus arcopallialis (RA), is not labeled (Figs. $4 A, 5 B, C$ ).

In contrast to the limited pallial expression, strong FoxP2 signal is detected in the avian striatum, striatum mediale (StM), and striatum laterale. Within the StM, the specific area required for song development, called area X (Sohrabji et al., 1990; Scharff and Nottebohm, 1991), expresses FoxP2 at a level comparable with or 
slightly higher than the surrounding regions (Fig. 3B, 5D). This strong striatal expression is consistent with the structural abnormalities observed in the caudate nucleus in affected humans (Watkins et al., 2002b; Belton et al., 2003). The globus pallidus (GP) is a major telencephalic component that exhibits sparsely distributed FoxP2 signals in the finch brain (Figs. $3 C$, $4 B, 5 C)$. It is worth noting that area $\mathrm{X}$ contains a pallidal component intermingled with the striatal one, and together these are proposed to comprise a pathway equivalent to the direct striato-pallido-thalamic pathway, mediated by globus pallidus interna (GPi) in the mammalian basal ganglia (Farries and Perkel, 2002). Therefore, FoxP2 signals within area $\mathrm{X}$ could include pallidal expression; however, our methods did not allow us to discriminate this. Congruent with this idea, human fetal GPi expresses FOXP2 (see below).

In the diencephalon, both dorsal and ventral thalamic structures strongly express FoxP2. The dorsal thalamic zone (DTZ) (Veenman et al., 1997), located dorsomedially in the avian diencephalon, shows distinct subregional labeling (Figs. $3 C, D, 5 A)$. The DTZ is homologous to the mammalian intralaminar, midline, and mediodorsal thalamic nuclear complex (IMMC) (Veenman et al., 1997). It consists of multiple nuclei with boundaries that likely underlie the observed pattern of FoxP2 expression. For example, nucleus dorsolateralis anterior thalami, pars medialis (DLM), part of the song circuit, expresses FoxP2 mRNA, as does dorsomedial thalamus, whereas nucleus dorsolateralis anterior thalami, pars lateralis does not (Fig. $3 C, D)$. Detailed immunohistochemical methods coupled with anterograde and retrograde tracing will be required for a more specific designation. In the vicinity of, but histologically distinct from, the DTZ is the ventrointermediate area (VIA), a region described in pigeons as comparable to the motor part of the mammalian ventral tier (Medina et al., 1997). In the zebra finch, FoxP2 signals are visible in this region just medial to the nucleus rotundus (Rt) (Fig. 3C).

A dorsal structure involved in visual processing, nucleus superficialis parvocellularis (Fig. 3D) (Trottier et al., 1995), is labeled. Other sensory thalamic nuclei with strong expression include ovoidalis (Ov), a major auditory input (Brauth and Reiner, 1991; Knudsen et al., 1993; Bruce et al., 2002), and Rt, which receives visual input from the tectum opticum (TeO) (Figs. 3C,D, 4D).

Sensory midbrain regions with substantial levels of FoxP2 include the auditory nucleus, mesencephalicus lateralis, pars dorsalis (MLd) (Fig. 5B), and TeO (Figs. 3D, 5A,B). Expression in mesencephalic motor regions includes label within the substantia nigra (Fig. $5 B$ ) and distributed label in the region containing the nucleus ruber (data not shown) (Wild et al., 1979). FoxP2 is expressed in the ventral tegmental area (Fig. 5A), which sends dopaminergic projections into area X (Lewis et al., 1981) and receives projections from the nucleus of the basal optic root. These nuclei are part of the accessory optic system in vertebrates, involved in multisensory analysis of self-motion (Wylie et al., 1999). In metencephalon, strong FoxP2 signals are observed in the vicinity of the nucleus reticularis pontis caudalis, pars gigantocellularis (Fig. 5B). These neurons are thought to play a role in the acoustic startle response and the sensorimotor integration of head-orienting movements (Nodal and Lopez, 2003; Park et al., 2003; Sasaki et al., 2004). In the cerebellum, Purkinje cells express FoxP2 (Fig. $5 B, D$ ). All FoxP2 signals were distributed symmetrically across hemispheres, as expected, given that lateralization of vocal control structures in songbirds is primarily peripheral (Suthers, 1997). Signals obtained with the second FoxP2 probe were identical to those obtained with the first (Fig. 4D) (see Materials and Methods) 

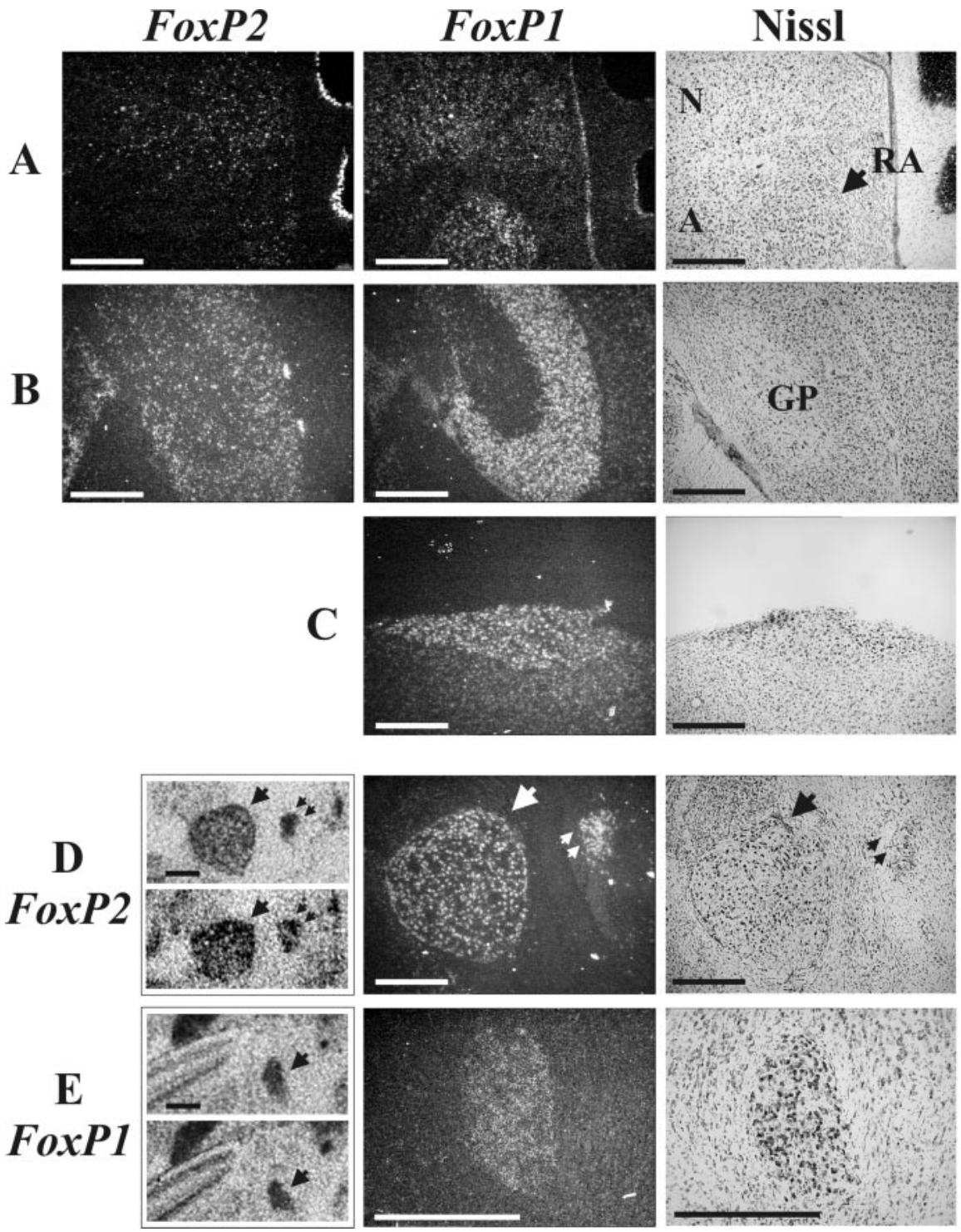

Figure 4. Representative magnified photomicrographs of selected regions of adult male zebra finch brain. In $A-C$, emulsiondipped material is shown next to the corresponding Nissl-stained section. $A$, FoxP2 signals are higher in the nidopallium (N) than in the arcopallium (A). The song nucleus RA, apparent in the Nissl stain (arrow), expresses FoxP1 signal but lacks FoxP2 signal. $B$, Images of the GP, recognizable in the Nissl-stained section, reveal the lack of FoxP1 and diffuse FoxP2 signals. $C$, The enhanced FoXP1 signal observed in the HVC with film autoradiography in Figures $3 C$ and $5, A$ and $C$, is confirmed with emulsion auotradiography and Nissl stain. D, E, Examples of subtelencephalic regions that express FoxP2 (small arrows, nucleus Ov; large arrow, nucleus Rt) and FoxP1 (nucleus SpL), respectively. In the left panels, film autoradiograms show the specificity of these expression patterns, confirmed by the use of two non-overlapping probes. In each, the top image corresponds to the first probe, and bottom to the second probe (see Materials and Methods). Remaining images are a higher magnification of the aforementioned structures shown with emulsion autoradiography (middle) and Nissl stain (right). Scale bars, $1 \mathrm{~mm}$.
(Fig. 5B). Of the remaining pallium, FoxP1 is strongly expressed in HD and the mesopallium (Figs. $3 A, B, 5 C, D$ ). Within the nidopallium, FoxP1 expression appears stronger rostral, versus caudal, to field L (Fig. 5D). There is a striking lack of signal in the vicinity of the nucleus basorostralis pallii (Bas) (Fig. $3 A, B$, arrowheads).

As in mouse lung (Shu et al., 2001; Lu et al., 2002), FoxP1 expression in zebra finch striatum and thalamus partially overlaps with that of FoxP2. Intriguingly, within the StM, area $\mathrm{X}$ is strongly labeled, above the level of the surrounding striatum (Figs. $3 B$, 5D). FoxP1 expression in area $\mathrm{X}$ is more evident than that of FoxP2, indicating that the stronger FoxP1 signal is not simply attributable to increased cell density but rather reflects enhanced expression in area $\mathrm{X}$. The GP appears to lack FoxP1 expression (Fig. $4 B$ ). Within the DTZ, FoxP1 expression overlaps with that of FoxP2, including within the DLM, the thalamic component of the song circuit (Fig. 3C,D). Unlike FoxP2, FoxP1 was not expressed in the sensory input nuclei Ov or Rt (Fig. $3 C, D)$. Whereas human brain imaging did not resolve specific structural thalamic deficits related to the human FOXP2 mutation, our zebra finch data indicate that the thalamus, in addition to the striatum, is another key site of FoxP1 and FoxP2 expression.

Mesencephalic FoxP1 signals, like those of FoxP2, occur in the aforementioned sensory regions MLd (Fig. $5 B$ ) and $\mathrm{TeO}$ (Figs. 3D, $4 E, 5 A, B$ ), although at lower levels. A visual processing nucleus, spiriformis lateralis (SpL) (Toledo et al., 2002), strongly expresses FoxP1 (Fig. 4 E). As with FoxP2, all FoxP1 signals were observed bilaterally, and patterns observed with the second probe were identical to those obtained with the first (Fig. 4E) (see Materials and Methods). In summary, both FoxP genes are expressed in visual and auditory nuclei critical for the sensory feedback required for song learning (Konishi, 1965; Morrison and Nottebohm, 1993), in addition to motor control regions.

\section{FoxP1 is expressed in song nuclei}

FoxP1 was investigated in addition to FoxP2, because it can dimerize with FoxP subfamily members (Wang et al., 2003; Li et al., 2004), shares a similar repressor domain, and can repress transcription from the same lung-specific promoters (Shu et al., 2001). Strikingly, FoxP1 shows expression within song circuit structures that are sexually dimorphic in zebra finches. In nidopallium, the song nucleus HVC shows enhanced expression (Figs. $3 C, 4 C, 5 A, C)$. Within the arcopallium, FoxP1 is clearly expressed in the song nucleus RA (Figs. $4 A, 5 B, C$ ) and slightly expressed ventrolateral to RA in the arcopallium dorsale (Ad)

\section{FoxP1 and FoxP2 in developing zebra finch brain}

As with many other Forkhead (Fox) transcription factors, Foxp1, Foxp2, and Foxp4 are implicated in organogenesis, specifically in lung and heart development (Shu et al., 2001; Lu et al., 2002). Recent studies of the KE family indicate that FoxP2 is critical for brain development (Lai et al., 2001) because its mutation leads to specific structural (Watkins et al., 2002b) and functional (Liegeois et al., 2003) neural deficits while apparently sparing the lungs and heart (Marcus and Fisher, 2003). We, therefore, examined both FoxP1 and FoxP2 expression in the developing brain of male zebra finches at $1 \mathrm{~d}$ after hatching (d1) and during the songlearning period at $\mathrm{d} 35$. At $\mathrm{d} 1$, the gross expression patterns of 


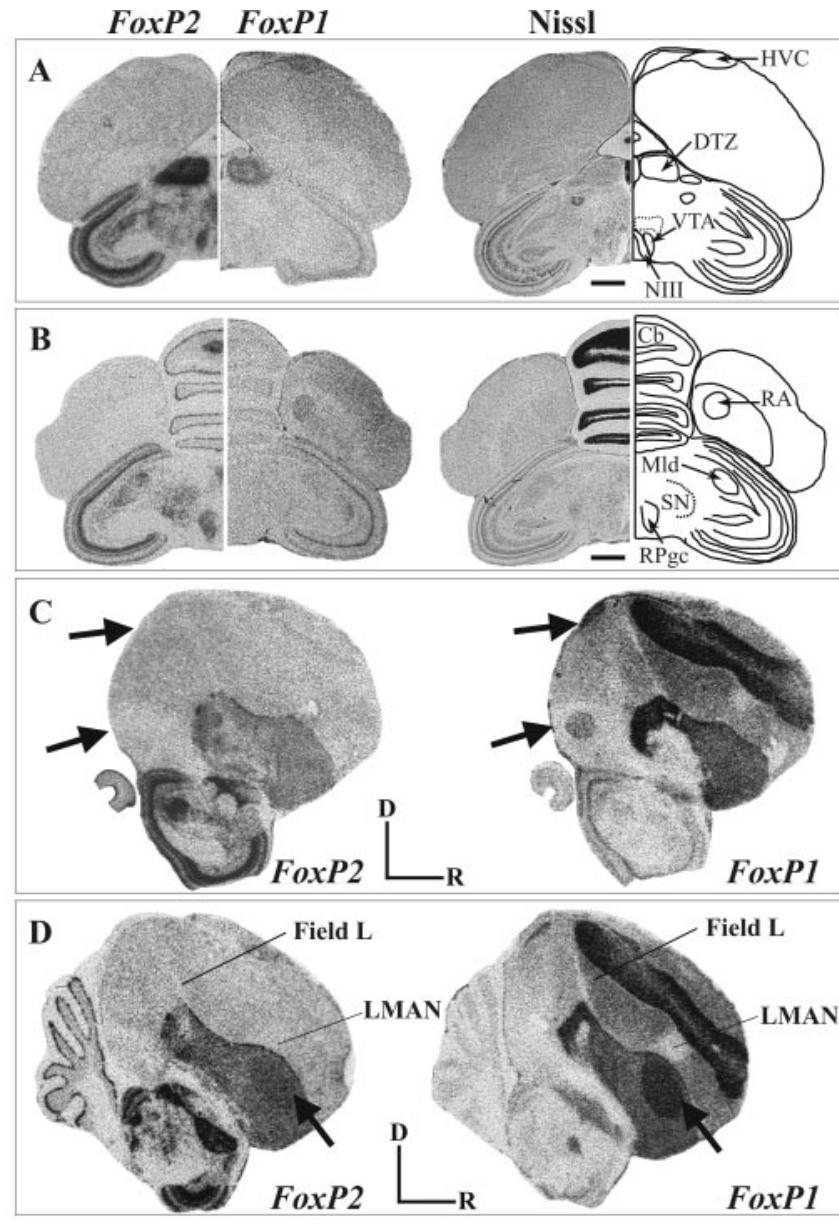

Figure 5. Representative bright-field photomicrographs of coronal $(A, B)$ or sagittal $(C, D)$ sections highlight (arrows) the enhanced expression of FoxP1 mRNA in song nuclei of adult male zebra finch brain, whereas no such enhancement is evident for FoxP2. $A, B$, Coronal images on the left half of the figure are of FoxP2 (far left) and FoxP1 expression patterns. On the right half of the figure, adjacent Nissl-stained sections and schematic drawings (far right) of these stains highlight enhanced areas of expression. Scale bars, $1 \mathrm{~mm}$. A, The premotor song nucleus HVC exhibits strong FoxP1 expression. FoxP2, in contrast, is only moderately expressed in the HVC at the level comparable with the surrounding nidopallium. $B, C$, The arcopallium, including RA, lacks FoxP2 signal. In contrast, FoxP1 is expressed in RA. D, The striatal song nucleus, area $X_{\text {, }}$ exhibits enhanced expression of FoxP1 while expressing FoxP2 at a level comparable with or slightly higher than the surrounding area. Both field $L$ and LMAN appear to lack FoxP signals. Note that the cerebellar expression of FoxP2 appears confined to Purkinje cells. The $5 \mathrm{~mm}$ scale bar represents dorsal (D) and rostral (R).

FoxP1 and FoxP2 resemble those in adults (Fig. 6A). Substantial FoxP1 mRNA is detected in regions that correspond to the pallium and striatum of adult telencephalon. FoxP2 is highly expressed in the striatum. Interestingly, strong signals are observed in regions lying near, but not directly adjacent to, the ventricle (data not shown), indicating a potential role for FoxP2 during migration or differentiation of neurons. In $\mathrm{d} 35$ brains, the specific expression patterns of FoxP1 and FoxP2 observed in adults are already evident, including strong expression of FoxP1 in the song nuclei, area X (Fig. 6B), HVC (Fig. 6F), and RA (data not shown) and subregional expression within the DTZ of both genes (Fig. 6C).

\section{Sites of sexual dimorphism}

Given the expression of FoxP1 and FoxP2 in many song nuclei and in sensory and motor pathways that are also crucial for malespecific song learning, we asked whether such expression patterns


Figure 6. Representative bright-field photomicrographs of developing zebra finch brains. Coronal sections demonstrate that the general expression patterns observed in adult brains for FoxP1 (right) and FoxP2 (left) mRNA are evident in younger animals. $A$, Bright-field images of sections through the head of $\mathrm{d} 1$ birds exhibit substantial FoxP1 expression in pallial regions and in the striatum. FoxP2 expression overlaps with that of FoxP1 in the striatum. Sections on the right were hybridized with the corresponding sense probes. $B, C, F$, In $\mathrm{d} 35$ male birds, characteristic expression patterns of $F$ ox $P 1$ and $F o x P 2$ are already evident, including enhanced expression of FoxP1 in the song nucleus, area $X(B)$, and HVC ( F). D, E, G, Images from d35 females reveal the sexually dimorphic expression of FoxP1, whereas FoxP2 lacks such dimorphism. Note the lack of enhanced expression of FoxP1 in the striatum, where area $X$ exists in males (compare $B, D$, right). The smaller female RA expresses FoxP1 ( $G$ ) (see Fig. $5 B$ for a comparison to adult male $R A)$.

are unique to male zebra finches. Our results reveal that FoxP2 expression does not differ consistently between sexes at juvenile or adult ages. For example, in d 35 females, FoxP2 mRNA is prominent in the striatum and the aforementioned regions of the dorsal thalamus (Fig. $6 D, E$ ). In some sections of $\mathrm{d} 35$ and adult male brains (Fig. $5 D$ ), the outline of area $\mathrm{X}$ was faintly discernible to the naked eye. However, this visibility was inconsistent between individual birds in both age groups. In sharp contrast, FoxP1 expression at $\mathrm{d} 35$ shows a consistent sexual dimorphism, concordant with the sexual dimorphism of the song circuit. In particular, in females, no enhancement of FoxP1 expression above the level of the surrounding striatum is detected in the StM, where the song nucleus, area $\mathrm{X}$, is present in males (Fig. 6, compare $B$, $D$ ). The FoxP1 riboprobe, however, detects mRNAs in the smaller RA of females as well as in adjacent Ad (Fig. 6G). More detailed investigation will determine whether any enhanced FoxP1 expression occurs in the smaller HVC of female zebra finches.

As stated above, the lack of FoxP2 sexual dimorphism reinforces our interpretation that dimorphic FoxP1 expression is not simply an epiphenomenon of cell density. Together, these findings suggest a role for FoxP1 as well as FoxP2 in the sexually dimorphic vocal learning of male zebra finches. We, thus, decided to investigate both FoxP1 and FoxP2 expression in humans.

FOXP1 and FOXP2 expression overlaps in subcortical regions of human fetal brain

Our data in the zebra finch, coupled with the articulation phenotype observed in affected members of the KE family, suggested to 

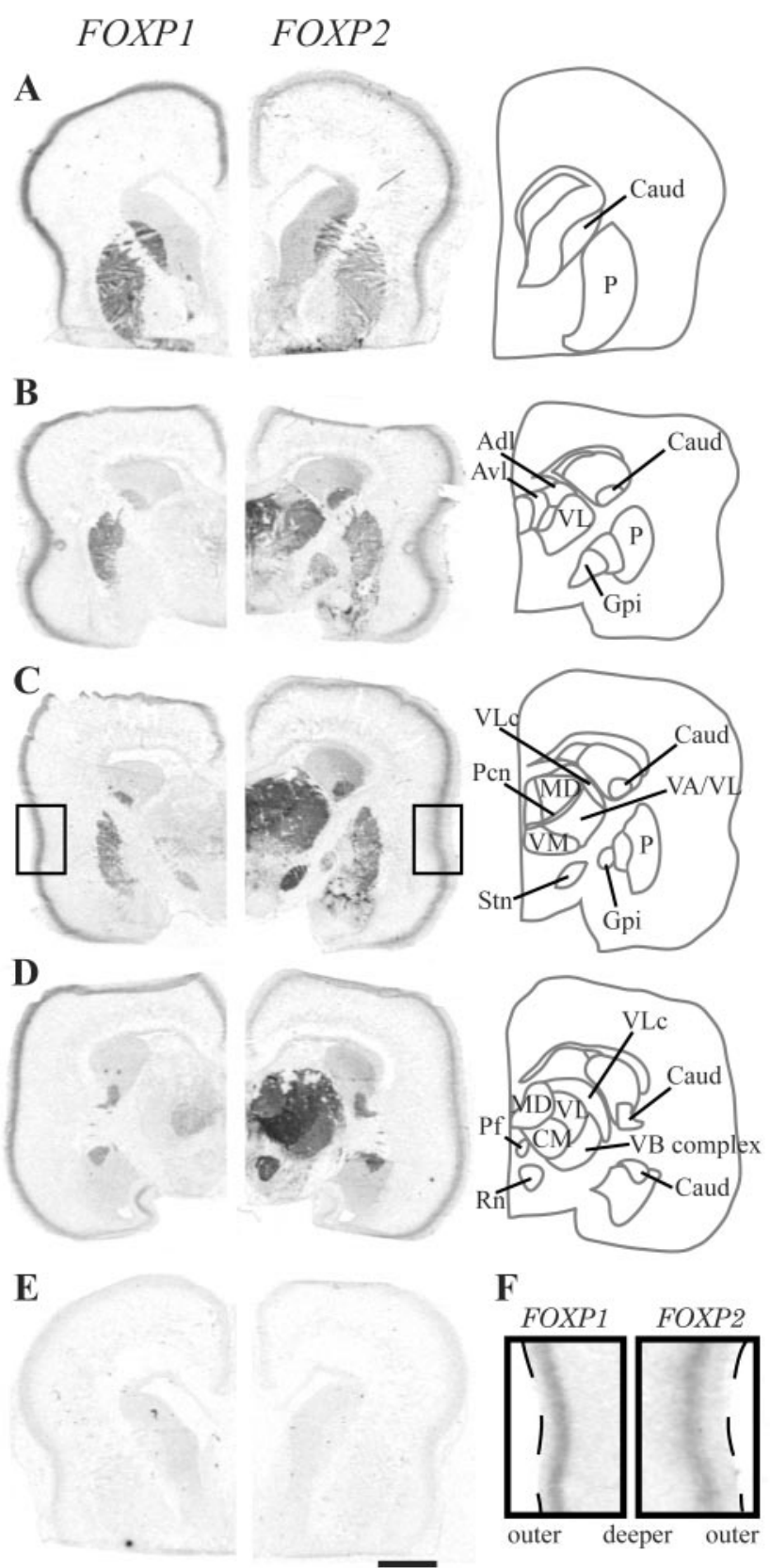

Figure 7. Representative bright-field photomicrographs of a series of coronal sections show regions of FOXP1 and FOXP2 mRNA expression in brains from 19 week $(A, E)$ and 22 week $(B-D)$ fetuses. Schematic figures based on Nissl-stained sections correspond to the adjacent photomicrographs on the right and highlight areas in which the FOXP genes are expressed. $A-D$, Sideby-side comparisons of FOXP1 (left) and FOXP2 (right) reveal nucleus caudatus and putamen with overlapping expressions of the two genes. $B-D, F O X P 2$ is expressed in thalamic structures of the somatic motor system. $E$, Sense probes for FOXP1 and FOXP2 present no significant signals. Scale bar: $A-F, 5 \mathrm{~mm}$. Magnification of the boxed areas in ( shows FOXP1 expression in the outer layers of the cortex and FOXP2 expression in the deeper cortical layers.

us that FOXP1 and FOXP2 expression patterns would be conserved between songbirds and humans. Specifically, the human language phenotype that arises from a mutation in FOXP2, coupled with the overlapping expression of FoxP2 with FoxP1 in the striatum and thalamus of the zebra finch, hints at a combinatorial role for these genes in the development of vocal control circuitry. This hypothesis would be supported by a similar overlap in the developing human brain. Thus, in situ hybridization analysis was performed between 15 (data not shown) and 22 weeks gestation
(Figs. 7, 8), when subcortical neurogenesis and migration are essentially complete and cortical neurogenesis is ongoing.

In the cortex, a complementary pattern of FOXP gene expression occurs in human embryos, with FOXP1 signals observed at more superficial layers than those of FOXP2 (Figs. 7C,E, $8 A$ ). Within the striatum, FOXP1 and FOXP2 are expressed in highly similar patterns, in the head and tail of nucleus caudatus and putamen (Figs. $7 A-D, 8 B$ ), where the intensity of FOXP label is reminiscent of the strong FoxP signals within the songbird striatum. Strikingly, FOXP2 shows restricted expression within the $\mathrm{GP}$, with high levels of expression in the GPi (Figs. $7 B, C, 8 B$ ), which provides the principal source of output from the basal ganglia to the nucleus centrum medianum thalami (CM) and the major motor relay nuclei of the thalamus. As in the zebra finch, human FOXP1 and FOXP2 expression overlaps in the thalamus, with FOXP2 revealing more extensive expression, specifically in the $\mathrm{CM}$ and nucleus medialis dorsalis thalami, both regions with homologs in the avian DTZ (Veenman et al., 1997) thalami (Fig. $7 C, D)$. More moderate signals arise from the nuclei anterior thalami, dorsal and ventral, and the nucleus parafascicularis thalami (Pf) (Fig. $7 B, D$ ). FOXP2 is expressed in the ventrobasal complex comprising the nucleus ventralis posterior lateralis/medialis (Fig. 7D). Similar to the VIA in the zebra finch (Medina et al., 1997), the ventral tier of the human thalamus exhibited strong FOXP2 expression, including nuclei ventralis anterior, lateralis, and posterior lateralis pars oralis (Fig. $7 C, D$ ). These nuclei have strong motor and premotor cortex connectivity, comprising key motor nuclei of the thalamus (Olszewski, 1952). Both genes also demonstrated significant expression in the nucleus subthalamicus bilaterally (Fig. 7C). Additionally, FOXP2 is strongly expressed in the nucleus ruber (Fig. 7D). The human brain regions of FOXP expression are key relays in essential motor control circuitry comprising premotor and posterior prefrontal pathways involved in motor planning and execution. This pattern of expression in specific subcortical structures for both FOXP1 and FOXP2 is entirely consistent with the putative role of these genes in pathways of sensorimotor integration that subserve vocalization and other complex learned motor movements. In no case did we observe asymmetry of FoxP gene expression.

\section{Discussion}

The discovery that FOXP2 is the monogenetic locus for a human language disorder affords the first opportunity to test a gene identified in the sole primate vocal learner, Homo sapiens, for its role in more experimentally accessible vocal learners, oscine songbirds. The corticostriatal abnormalities and speech disruption observed in humans bearing a FOXP2 mutation, coupled with the well-described corticostriatal song circuit in songbirds, suggested the exciting hypothesis that FoxP2 regulates common mechanisms for vocal learning. As a first test of this hypothesis, we identified the full-length homolog of FOXP2 in the zebra finch, an oscine songbird. The deduced amino acid FoxP2 sequence in the finch (Fig. 2) does not contain the two residues that, among primates, are specific to humans. However, it does possess five residues that differ from currently known mammalian forms. This finding sustains the possibility that among mammals and among birds, independent variation in FoxP2 secondary structure contributed to the capacity for vocal learning in certain species (Enard et al., 2002; Zhang et al., 2002; Clark et al., 2003).

Before the identification of the KE family mutation, research on Foxp2 focused on the lung airway epithelium, where Foxp1, Foxp2, and Foxp4 exhibit coordinate developmental expression 
with regions of distinction as well as overlap (Shu et al., 2001; Lu et al., 2002). Here, in the brains of an avian and a primate vocal learner, we find that FoxP1 and FoxP2 have both distinct and shared expression patterns in the cortex, striatum, and thalamus. Based on the human mutant phenotype, we predicted FoxP expression within the song circuit of the male zebra finch, a species in which only males learn to sing a courtship song. We find that FoxP2 is indeed expressed within area $\mathrm{X}$ of the striatum; however, this expression is not sexually dimorphic. In sharp contrast, FoxP1 exhibits several regions of sexual dimorphism. In two song nuclei, HVC and area $\mathrm{X}$, FoxP1 expression overlaps with that of FoxP2 and is higher than in the surrounding regions. Expression within area $\mathrm{X}$ is of particular interest because this nucleus is the specific region of the striatum required for song development (Sohrabji et al., 1990; Scharff and Nottebohm, 1991) and that exhibits song-selective neuronal responses during singing (Jarvis and Nottebohm, 1997) or playback (Solis and Doupe, 2000) of the bird's own song.

In regions of overlap, FoxP1 and FoxP2 could act as coregulators in the brain, as indicated in the lung (Shu et al., 2001) and by the capacity for Foxp1 to dimerize with other subfamily members (Wang et al., 2003; Li et al., 2004). In zebra finches, FoxP1 expression could confer a sexually dimorphic function on FoxP2 in sites where dimorphic FoxP1 expression overlaps with monomorphic FoxP2. The monomorphic expression in brain regions of song nuclei raises two possibilities. First, it could reflect the potential of females to learn to sing when given early hormonal treatment (Gurney and Konishi, 1980; Akutagawa and Konishi, 2001; Grisham et al., 2002) and, more generally, for females of other songbird species to sing. Simply put, a given FoxP molecule may be necessary, but not sufficient, for vocal learning and likely interacts with other proteins for that potential to be realized. In this regard, neural expression patterns of all FoxP subfamily members across avian phylogeny will be informative (Haesler et al., 2004). Second, monomorphic expression between male and female zebra finches in song control regions may highlight areas of sensory processing used by both sexes in perception of song (Brenowitz, 1991; MacDougallShackelton et al., 1998; Leitner and Catchpole, 2002).

Our results in the finch predicted that in humans, FOXP1, in addition to FOXP2, would be expressed in similar cortical, striatal, and thalamic patterns. The avian pallium bears homology to the mammalian cortex, however, a one-to-one correspondence between cortical and pallial layers is lacking (Reiner et al., 2004). Despite this structural difference, the cortical complementarity of human FOXP expression is reminiscent of that in the zebra finch pallium, where FoxP2 is diffusely expressed whereas robust FoxP1 expression is localized to the HD, mesopallium, and the song nucleus HVC. In line with our prediction, FoxP1 and FoxP2 expression overlaps in both songbird and human striatum, in-
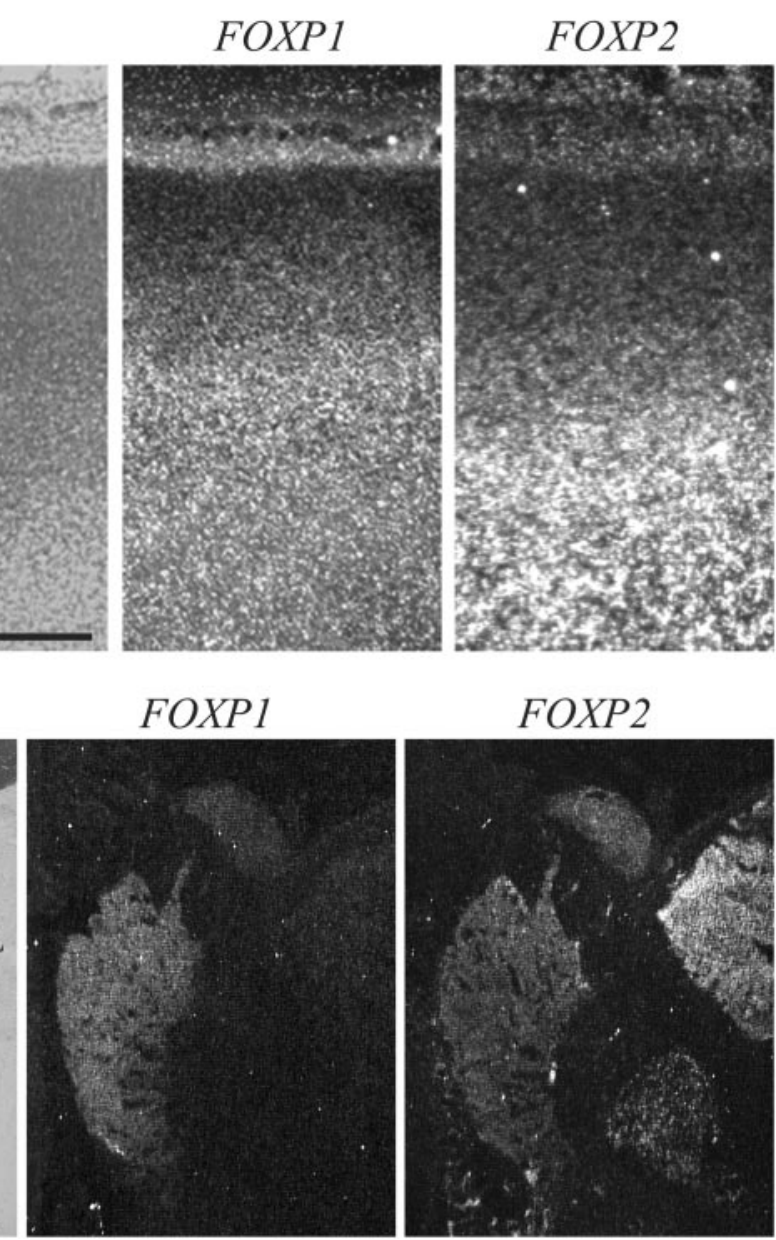

Figure 8. Dark-field images from emulsion autoradiography (right) alongside corresponding bright-field images of Nisslstained sections (left). A, FOXP1 shows expression in the cortical plate ranging from layers II/III and deeper. FOXP2 shows pronounced expression in layer VI and the subplate and the intermediate zone. B, FOXP2 has expression in the GPi, where silver grains are absent for FOXP1 expression. FOXP2 also shows stronger expression in VL than FOXP1. Scale bars: $A, 0.5 \mathrm{~mm} . B, 1 \mathrm{~mm}$.

cluding within songbird area $\mathrm{X}$ and in the human nucleus caudatus and putamen. To the extent that mammalian and avian thalamic subregions have been compared (Medina et al., 1997; Veenman et al., 1997), FoxP gene expression is quite similar in human and songbird. This includes strong expression in motor structures within the songbird DTZ and in the vicinity of the VIA and the human IMMC and ventral tier. Sensory thalamus, in addition to motor thalamus, also expresses FoxP mRNA. Similarly, in mesencephalon, both sensory and motor structures are labeled in humans and songbirds. This expression pattern is compatible with a role for these molecules in movements that rely on sensory feedback (Konishi, 1965; Morrison and Nottebohm, 1993).

Interestingly, FoxP genes are expressed in regions of the zebra finch DTZ that are homologous to the CM and Pf in mammals (Veenman et al., 1997; Bruce et al., 2002). These thalamic nuclei are hypothesized to provide "attention-specific sensory information important for conditioned responses" in primates (Sidibe et al., 2002). In rats, Pf appears important for orofacial function (Tsumori et al., 2002, 2003). Yet, the Bas of the songbird, an orofacial control region implicated in feeding (Wild and Farabaugh, 1996), conspicuously lacks FoxP1 (Fig. 3A,B). This example again points to roles for FoxP molecules in learned, rather than purely innate, orofacial behaviors, a hypothesis that 
invites further study. One hint from the human behavioral data is that affected members of the KE family display no problem with musical pitch and intonation, yet are impaired on production and perception of rhythms (Alcock et al., 2000b). This central deficit in temporal patterning is consistent with a role for FoxP molecules in tasks that require sensory feedback to trigger or predict motor output.

The lack of asymmetry in human FOXP1 and FOXP2 neural expression is not surprising given the bilateral nature of the structural deficits observed in affected KE family members (Belton et al., 2003). In both humans and songbirds, FoxP expression occurs early in development, at a time when both hemispheres of the human brain are capable, albeit not equipotent, of giving rise to language later in development (MacWhinney et al., 2000; Vicari et al., 2000). Given the bilateral expression of the FoxP genes observed here, molecular understanding of the predominantly lateralized aspects of language function in humans remains as a significant challenge for future studies.

Our findings provide the first view of FOXP1 neural expression in humans. Furthermore, they complement and extend recent studies on FOXP2 expression in mammalian brain, because the human developmental stage examined here allows for finer localization to subcortical structures than in prior studies (Lai et al., 2003). Although we have focused on FOXP mRNA, findings in mouse (Ferland et al., 2003) and zebra finch (Haesler et al., 2004) brain indicate that mRNA and protein are localized similarly. The emergent pattern for FOXP2 is of robust cortical, basal ganglia, thalamic, and cerebellar expression. The complementary cortical expression of FOXP1 versus FOXP2 found in humans is similar to that in mouse (Ferland et al., 2003). An interesting difference is the discovery that human GPi expresses FOXP2 (Fig. $6 C$ ), because no such signal was detected in mouse (Ferland et al., 2003) and GPi was not previously reported on in human (Lai et al., 2003). This result provides additional support to a motor role for FOXP2, because GPi is the principal source of output from the basal ganglia to the major motor relay nuclei of the thalamus in humans.

Our findings, together with those of Haesler et al. (2004), provide the first picture of FoxP neural expression in another vocal learner, an oscine songbird, and do so across developmental time points. Persistent expression in adult zebra finches may indicate additional roles in the mature brain because other striatal transcription factors tend to exhibit developmental downregulation (Takahashi et al., 2003). Because the timeline and the specific structures for song learning are known, songbird research can further define the pathways for vocal learning that FoxP2 acts on and to discover new molecules that may be common to vocal learning (Clayton et al., 1988; Denisenko-Nehrbass et al., 2000; Akutagawa and Konishi, 2001), including FoxP1. In the zebra finch, the high levels of FoxP2 in striatum and thalamus, coupled with sexually dimorphic expression of FoxP1 in multiple song control regions including area $\mathrm{X}$, may reflect combinatorial regulation by these proteins of the development of vocal control structures. Taken together, the similar patterns of FoxP gene expression in zebra finch and human suggest that FoxP1, in addition to FoxP2, is likely to play an important role in the formation and function of circuits for learned articulation requiring fine sequential motor control in songbird and human.

\section{References}

Akutagawa E, Konishi M (2001) A monoclonal antibody specific to a song system nuclear antigen in estrildine finches. Neuron 31:545-556.
Alcock KJ, Passingham RE, Watkins KE, Vargha-Khadem F (2000a) Oral dyspraxia in inherited speech and language impairment and acquired dysphasia. Brain Lang 75:17-33.

Alcock KJ, Passingham RE, Watkins K, Vargha-Khadem F (2000b) Pitch and timing abilities in inherited speech and language impairment. Brain Lang 75:34-46.

Belton E, Salmond CH, Watkins KE, Vargha-Khadem F, Gadian DG (2003) Bilateral brain abnormalities associated with dominantly inherited verbal and orofacial dyspraxia. Hum Brain Mapp 18:194-200.

Bottjer SW, Johnson F (1997) Circuits, hormones, and learning: vocal behavior in songbirds. J Neurobiol 33:602-618.

Bottjer SW, Miesner EA, Arnold AP (1984) Forebrain lesions disrupt development but not maintenance of song in passerine birds. Science 224:901-903.

Bottjer SW, Halsema KA, Brown SA, Miesner EA (1989) Axonal connections of a forebrain nucleus involved with vocal learning in zebra finches. J Comp Neurol 279:312-326.

Brainard MS, Doupe AJ (2000) Interruption of a basal ganglia-forebrain circuit prevents plasticity of learned vocalizations. Nature 762-766.

Brauth SE, Reiner A (1991) Calcitonin-gene related peptide is an evolutionarily conserved marker within the amniote thalamo-telencephalic auditory pathway. J Comp Neurol 313:227-239.

Brenowitz EA (1991) Altered perception of species-specific song by female birds after lesions of a forebrain nucleus. Science 251:303-305.

Bruce LL, Kornblum HI, Seroogy KB (2002) Comparison of thalamic populations in mammals and birds: expression of ErbB4 mRNA. Brain Res Bull 57:455-461.

Carlsson P, Mahlapuu M (2002) Forkhead transcription factors: key players in development and metabolism. Dev Biol 250:1-23.

Clark AG, Glanowski S, Nielsen R, Thomas PD, Kejariwal A, Todd MA, Tanenbaum DM, Civello D, Lu F, Murphy B, Ferriera S, Wang G, Zheng X, White TJ, Sninsky JJ, Adams MD, Cargill M (2003) Inferring nonneutral evolution from human-chimp-mouse orthologous gene trios. Science 302:1960-1963.

Clayton DF, George JM (1999) Synucleins in synaptic plasticity and neurodegenerative disorders. J Neurosci Res 58:120-129.

Clayton DF, Huecas ME, Sinclair-Thompson EY, Nastiuk KL, Nottebohm F (1988) Probes for rare mRNAs reveal distributed cell subsets in canary brain. Neuron 1:249-261.

Denisenko-Nehrbass NI, Jarvis E, Scharff C, Nottebohm F, Mello CV (2000) Site-specific retinoic acid production in the brain of adult songbirds. Neuron 27:359-370.

Doupe AJ, Kuhl PK (1999) Birdsong and human speech: common themes and mechanisms. Annu Rev Neurosci 22:567-631.

Enard W, Przeworski M, Fisher SE, Lai CSL, Wiebe V, Kitano T, Monaco AP, Paeaebo S (2002) Molecular evolution of FOXP2, a gene involved in speech and language. Nature 418:869-872.

Farries MA (2001) The oscine song system considered in the context of the avian brain: lessons learned from comparative neurobiology. Brain Behav Evol 58:80-100.

Farries MA, Perkel DJ (2002) A telencephalic nucleus essential for song learning contains neurons with physiological characteristics of both striatum and globus pallidus. J Neurosci 22:3776-3787.

Feess-Higgins A, Larroche J (1987) Development du cerveau foetal humain: atlas anatomique. Paris: INSERM Masson.

Ferland RJ, Cherry TJ, Preware PO, Morrisey EE, Walsh CA (2003) Characterization of Foxp2 and Foxp1 mRNA and protein in the developing and mature brain. J Comp Neurol 460:266-279.

Fisher SE, Lai CS, Monaco AP (2003) Deciphering the genetic basis of speech and language disorders. Annu Rev Neurosci 26:57-80.

Geschwind DH, Ou J, Easterday MC, Dougherty JD, Jackson RL, Chen Z, Antoine H, Terskikh A, Weissman IL, Nelson SF, Kornblum HI (2001) A genetic analysis of neural progenitor differentiation. Neuron 29:325-339.

Grisham W, Lee J, McCormick ME, Yang-Stayner K, Arnold AP (2002) Antiandrogen blocks estrogen-induced masculinization of the song system in female zebra finches. J Neurobiol 51:1-8.

Gurney ME, Konishi M (1980) Hormone-induced sexual differentiation of brain and behavior in zebra finches. Science 208:1380-1383.

Haesler S, Wada K, Nshdejan A, Morrisey EE, Lints T, Jarvis ED, Scharff C (2004) FoxP2 expression in avian vocal learners and non-learners. J Neurosci 24:3164-3175. 
Jacobs EC, Arnold AP, Campagnoni AT (1999) Developmental regulation of the distribution of aromatase- and estrogen-receptor- mRNAexpressing cells in the zebra finch brain. Dev Neurosci 21:453-472.

Jarvis ED, Nottebohm F (1997) Motor-driven gene expression. Proc Natl Acad Sci USA 94:4097-4102.

Jones EG (1985) The thalamus. New York: Plenum.

Kaestner KH, Knochel W, Martinez DE (2000) Unified nomenclature for the winged helix/forkhead transcription factors. Genes Dev 14:142-146.

Knudsen EI, Knudsen PF, Masino T (1993) Parallel pathways mediating both sound localization and gaze control in the forebrain and midbrain of the barn owl. J Neurosci 13:2837-2852.

Konishi M (1965) The role of auditory feedback in the control of vocalization in the white-crowned sparrow. Zeitschrift fur Tierpsychologie 22:770-783.

Lai CS, Gerrelli D, Monaco AP, Fisher SE, Copp AJ (2003) FOXP2 expression during brain development coincides with adult sites of pathology in a severe speech and language disorder. Brain 126:2455-2462.

Lai CSL, Fisher SE, Hurst JA, Vargha-Khadem F, Monaco AP (2001) A forkhead-domain gene is mutated in a severe speech and language disorder. Nature 413:519-523.

Leitner S, Catchpole CK (2002) Female canaries that respond and discriminate more between male songs of different quality have a larger song control nucleus (HVC) in the brain. J Neurobiol 52:294-301.

Lewis JW, Ryan SM, Arnold AP, Butcher LL (1981) Evidence for a catecholaminergic projection to area $\mathrm{X}$ in the zebra finch. J Comp Neurol 196:347-354.

Li S, Weidenfeld J, Morrisey EE (2004) Transcriptional and DNA binding activity of the Foxp1/2/4 family is modulated by heterotypic and homotypic protein interactions. Mol Cell Biol 24:809-822.

Liegeois F, Baldeweg T, Connelly A, Gadian DG, Mishkin M, Vargha-Khadem F (2003) Language fMRI abnormalities associated with FOXP2 gene mutation. Nat Neurosci 6:1230-1237.

Lu MM, Li S, Yang H, Morrisey EE (2002) Foxp4: a novel member of the Foxp subfamily of winged-helix genes co-expressed with Foxp1 and Foxp2 in pulmonary and gut tissues. Gene Expr Patterns 2:223-228.

MacDougall-Shackelton SA, Hulse SH, Ball GF (1998) Neural bases of song preferences in female zebra finches (Taeniopygia guttata). NeuroReport 9:3047-3052.

MacWhinney B, Feldman H, Sacco K, Valdes-Perez R (2000) Online measures of basic language skills in children with early focal brain lesions. Brain Lang 71:400-431.

Marcus GF, Fisher SE (2003) FOXP2 in focus: what can genes tell us about speech and language? Trends Cogn Sci 7:257-262.

Medina L, Veenman CL, Reiner A (1997) Evidence for a possible avian dorsal thalamic region comparable to the mammalian ventral anterior, ventral lateral, and oral ventroposterolateral nuclei. J Comp Neurol 384:86-108.

Mooney R (2000) Different subthreshold mechanisms underlie song selectivity in identified HVc neurons of the zebra finch. J Neurosci 20:5420-5436.

Mooney R, Konishi M (1991) Two distinct inputs to an avian song nucleus activate different glutamate receptor subtypes on individual neurons. Proc Natl Acad Sci USA 88:4075-4079.

Morrison RG, Nottebohm F (1993) Role of a telencephalic nucleus in the delayed song learning of socially isolated zebra finches. J Neurobiol 24:1045-1064.

Nodal FR, Lopez DE (2003) Direct input from cochlear root neurons to pontine reticulospinal neurons in albino rat. J Comp Neurol 460:80-93.

Nottebohm F, Arnold AP (1976) Sexual dimorphism in vocal control areas of the songbird brain. Science 194:211-213.

Nottebohm F, Stokes TM, Leonard CM (1976) Central control of song in the canary, Serinus canarius. J Comp Neurol 165:457-486.

Nottebohm F, Kelley DB, Paton JA (1982) Connections of vocal control nuclei in the canary telencephalon. J Comp Neurol 207:344-357.

O'Garra A, Vieira P (2003) Twenty-first century Foxp3. Nat Immunol 4:304-306.

Okuhata S, Saito N (1987) Synaptic connections of thalamo-cerebral vocal nuclei of the canary. Brain Res Bull 18:35-44.

Olszewski J (1952) The thalamus of the Macaca mulatta: an atlas for use with the stereotaxic instrument. New York: Karger.

Packard MG, Knowlton BJ (2002) Learning and memory functions of the basal ganglia. Annu Rev Neurosci 25:563-593.
Park IK, Kim MK, Tomoro I, Masato U (2003) A study on the spinoreticulocerebellar tract in chickens. J Vet Sci 4:1-8.

Perlman WR, Ramachandran B, Arnold AP (2003) Expression of androgen receptor mRNA in the late embryonic and early posthatch zebra finch brain. J Comp Neurol 455:513-530.

Reiner A, Perkel DJ, Bruce L, Butler AB, Csillag A, Kuenzel W, Medina L, Paxinos G, Shimizu T, Striedter G, Wild M, Ball GF, Durand S, Güntürkün O, Lee DW, Mello CV, Powers A, White SA, Hough G, Kubikova L, Smulders TV, Wada K, Dugas-Ford J, Husband S, Yamamoto K, Yu J, Siang C, Jarvis ED (2004) Revised nomenclature for avian telencephalon and some related brainstem nuclei. J Comp Neurol, in press.

Sasaki S, Yoshimura K, Naito K (2004) The neural control of orienting: role of multiple-branching reticulospinal neurons. Prog Brain Res 143:383-389.

Scharff C, Nottebohm F (1991) A comparative study of the behavioral deficits following lesions of various parts of the zebra finch song system: implications for vocal learning. J Neurosci 11:2896-2913.

Shu WG, Yang HH, Zhang LL, Lu MM, Morrisey EE (2001) Characterization of a new subfamily of winged-helix/forkhead (Fox) genes that are expressed in the lung and act as transcriptional repressors. J Biol Chem 276:27488-27497.

Sidibe M, Pare JF, Smith Y (2002) Nigral and pallidal inputs to functionally segregated thalamostriatal neurons in the centromedian/parafascicular intralaminar nuclear complex in monkey. J Comp Neurol 447:286-299.

Snowdon C, Hausberger M (1997) Social influences on vocal development. Cambridge, UK: Cambridge UP.

Sohrabji F, Nordeen EJ, Nordeen KW (1990) Selective impairment of song learning following lesions of a forebrain nucleus in the juvenile zebra finch. Behav Neural Biol 53:51-63.

Solis MM, Doupe AJ (2000) Compromised neural selectivity for song in birds with impaired sensorimotor learning. Neuron 25:109-121.

Stokes TM, Leonard CM, Nottebohm F (1974) The telencephalon, diencephalon, and mesencephalon of the canary, Serinus canaria, in stereotaxic coordinates. J Comp Neurol 156:337-374.

Suthers RA (1997) Peripheral control and lateralization of birdsong. J Neurobiol 33:632-652.

Takahashi K, Liu FC, Hirokawa K, Takahashi H (2003) Expression of Foxp2, a gene involved in speech and language, in the developing and adult striatum. J Neurosci Res 73:61-72.

Toledo CA, Pezzini R, Santos RC, Britto LR (2002) Expression of AMPAtype glutamate receptors in pretectal nuclei of the chick brain. Brain Res Bull 57:359-361.

Tolivia J, Tolivia D (1985) A new technique for differential and simultaneous staining of nerve cells and fibers. J Neurosci Methods 13:305-311.

Trottier C, Reperant J, Miceli D (1995) Anatomical evidence of a retinothalamo-hippocampal pathway in the pigeon (Columba livia). J Hirnforsch 36:489-500.

Tsumori T, Yokota S, Ono K, Yasui Y (2002) Synaptic organization of GABAergic projections from the substantia nigra pars reticulata and the reticular thalamic nucleus to the parafascicular thalamic nucleus in the rat. Brain Res 957:231-241.

Tsumori T, Yokota S, Ono K, Yasui Y (2003) Nigrothalamostriatal and nigrothalamocortical pathways via the ventrolateral parafascicular nucleus. NeuroReport 14:81-86.

Vargha-Khadem F, Watkins K, Alcock K, Fletcher P, Passingham R (1995) Praxic and nonverbal cognitive deficits in a large family with a genetically transmitted speech and language disorder. Proc Natl Acad Sci USA 92:930-933.

Vargha-Khadem F, Watkins KE, Price CJ, Ashburner J, Alcock KJ, Connelly A, Frackowiak RSJ, Friston KJ, Pembrey ME, Mishkin M, Gadian DG, Passingham RE (1998) Neural basis of an inherited speech and language disorder. Proc Natl Acad Sci USA 95:12695-12700.

Vates GE, Nottebohm F (1995) Feedback circuitry within a song-learning pathway. Proc Natl Acad Sci USA 92:5139-5143.

Veenman CL, Medina L, Reiner A (1997) Avian homologues of mammalian intralaminar, mediodorsal and midline thalamic nuclei: immunohistochemical and hodological evidence. Brain Behav Evol 49:78-98.

Vicari S, Albertoni A, Chilosi AM, Cipriani P, Cioni G, Bates E (2000) Plasticity and reorganization during language development in children with early brain injury. Cortex 36:31-46.

Wang B, Lin D, Li C, Tucker P (2003) Multiple domains define the expression and regulatory properties of Foxp 1 forkhead transcriptional repressors. J Biol Chem 278:24259-24268. 
Watkins KE, Dronkers NF, Vargha-Khadem F (2002a) Behavioural analysis of an inherited speech and language disorder: comparison with acquired aphasia. Brain 125:452-464.

Watkins KE, Vargha-Khadem F, Ashburner J, Passingham RE, Connelly A, Friston KJ, Frackowiak RS, Mishkin M, Gadian DG (2002b) MRI analysis of an inherited speech and language disorder: structural brain abnormalities. Brain 125:465-478.

Wild JM (1993) The avian nucleus retroambigualis: a nucleus for breathing, singing and calling. Brain Res 606:319-324.

Wild JM, Farabaugh SM (1996) Organization of afferent and efferent projections of the nucleus basalis prosencephali in a passerine, Taeniopygia guttata. J Comp Neurol 365:306-328.
Wild JM, Cabot JB, Cohen DH, Karten HJ (1979) Origin, course and terminations of the rubrospinal tract in the pigeon (Columba livia). J Comp Neurol 187:639-654.

Williams H, Mehta N (1999) Changes in adult zebra finch song require a forebrain nucleus that is not necessary for song production. J Neurobiol $39: 14-28$.

Wylie DR, Glover RG, Aitchison JD (1999) Optic flow input to the hippocampal formation from the accessory optic system. J Neurosci 19:5514-5527.

Zhang J, Webb DM, Podlaha O (2002) Accelerated protein evolution and origins of human-specific features: Foxp2 as an example. Genetics 162: 1825-1835. 